NASA Technical Memorandum 101328

\title{
High Frequency GaAlAs Modulator and Photodetector for Phased Array Antenna Applications
}

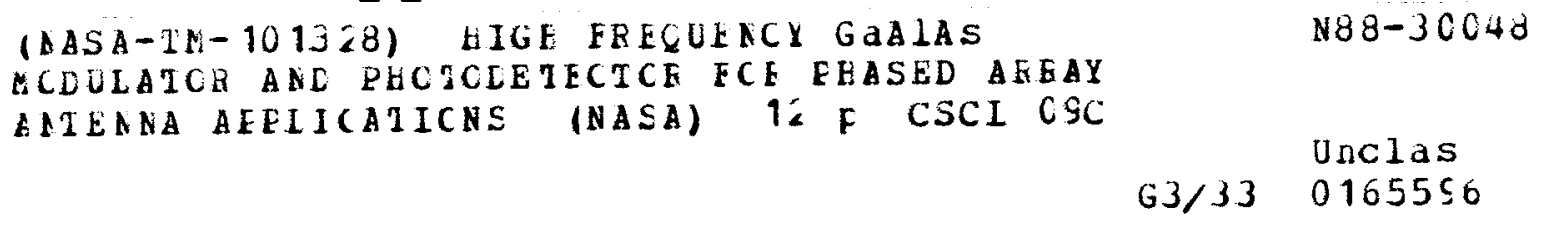

N88-30048

Unclas G3/33 $01655 \varsigma_{6}$

P.C. Claspy, C.M. Chorey, and S.M. Hill Case Western Reserve University

Cleveland, Ohio

and

K.B. Bhasin

Lewis Research Center

Cleveland, Ohio

Prepared for the International Symposium and Exhibition on Fiber Optics, Optoelectronics, and Laser Applications sponsored by the Society of Photo-Optical Instrumentation Engineers Boston, Massachusetts, September 6-9, 1988 
HIGH FREQUENCY GaAIAS MODULATOR AND PHOTODETECTOR FOR PHASED ARRAY ANTENNA APPLICATIONS

\author{
P.C. Claspy, C.M. Chorey, and S.M. Hill
}

Case Western Reserve University, Department of Electrical Engineering and Applied Physics Cleveland, Ohio 44106

and

K. B. Bhasin

National Aeronautics and Space Administration, Lewis Research Center, Cleveland, Ohio 44135

\title{
ABSTRACT
}

A waveguide Mach-Zehnder electro-optic modulator and an interdigitated photoconductive detector designed to operate at $820 \mathrm{~nm}$, fabricated on different GaAlAs/GaAs heterostructure materials, are being investigated for use in optical interconnects in phased array antenna systems. Measured optical attenuation effects in the modulator are discussed and the observed modulation performance up to $1 \mathrm{GHz}$ is presented. Measurements of detector frequency response are described and results of these measurements are presented.

\section{INTRODUCTION AND BACKGROUND}

Considerable attention has been devoted over the past few years to the use of monolithic microwave integrated circuits (MMIC's) in phased array antenna systems. Although phased arrays consisting of hundreds of elements are becoming a reality, questions regarding practical interconnection methods for information and control signal distribution to these elements remain.1.2 Waveguide, coaxial cable, and microstrip transmission lines suffer from various combinations of high weight, mechanical inflexibility, and high loss, whereas fiber optic interconnects have the potential to overcome these limitations if various technological issues can be successfully addressed. Among these issues are the efficient high speed modulation of the output of semiconductor lasers, and the efficient detection and demodulation of that light at the array element. To enhance the usefulness of modulators and detectors in the proposed MMIC application they should be chip-level integrable with the GaAlAs/GaAs heterostructure devices, such as high electron mobility transistors (HEMT's), that have been demonstrated to have a significant speed advantage over MESFET-type devices in MMIC's.

High speed modulation of the output of a diode laser can be achieved either by direct modulation of the driving current or by use of an external device such as an electro-optic modulator. Significant successes using the former approach have been reported, 3 and directly modulated lasers with modulation bandwidths in the 10 to $20 \mathrm{GHz}$ range are commercially available at both $820 \mathrm{~nm}$ and $1.33 \mu \mathrm{m.4}$ Various authors have also reported external electrooptic modulators based on $\mathrm{LiNbO}_{3}{ }^{5}$ and on GaAlAs/GaAs. ${ }^{6}$ The $\mathrm{LiNbO}_{3}$ devices are not chip level integrable with semiconductor lasers using current technology, and most of the research on GaAlAs/GaAs waveguide modulators has been at $1.33 \mu \mathrm{m}$.

Numerous authors have also described high speed GaAs-based optical detectors, including a Schottky photodiode that is useful to $100 \mathrm{GHz}, 7$ and GaAlAs/GaAs-based detectors have been reported.8,9 At the onset of our research, however, no systematic study of the effects of material and device geometry on the performance of the latter detector type had been reported.

In this paper we discuss the results obtained to date on the performance of a triple heterostructure GaAlAs traveling wave electro-optic modulator and of a series of interdigitated photoconductive detectors fabricated on a HEMT-type heterostructure material. An objective of our current research is to investigate detectors and external modulators that operate at $820 \mathrm{~nm}$ and which are potentially easily integrated with HEMT-type MMIC's. The modulator is a waveguide Mach-Zehnder device that is driven by a $50 \Omega$ coplanar electrode structure. Detailed discussions of the design and fabrications of the modulator have been presented previously, 10-12 so in this paper we address primarily the observed performance and an analysis of those results. The detectors, on the other hand, have not been described previously, so the discussion will encompass their design and fabrication as well as their performance. In section 2, the configuration and performance of the modulator is discussed, Section 3 describes the design and fabrication of the detectors, and section 4 presents an 
overview of the observed detector performance. Finally, in section 5 the results will be summarized and concluding remarks will be made.

\section{MODULATOR CHARACTERISTICS AND PERFORMANCE}

The electro-optic modulator, shown schematically in Fig. 1, consists of two features, namely, the strip-loaded, single-mode optical waveguide Mach-Zehnder interferometer, and the coplanar waveguide electrodes. As shown in Fig. 2, the three layers of the MBE-grown material are GaAlAs, with the concentrations of Al chosen to shift the absorption edge to shorter wavelengths while still providing adequate guiding. The upper layer was etched to form the loading ridge, and the optical signal propagates in the middle layer. This configuration concentrates the light energy away from the etched edges of the loading ridge to reduce the light scattering. To prevent additional losses the first layer above the semiinsulating GaAs was chosen to be thick enough that the evanescent optical field does not extend to the substrate. A coplanar traveling wave electrode structure was chosen to provide the microwave field in the required direction. It was designed for $50 \Omega$ impedance on semi-insulating GaAs and it was assumed, since there is no doping in any layer and the Al fraction is small, that the design remains valid when implemented on GaAlAs. The calculated half-wave voltage is $18 \mathrm{~V}$ and the $3 \mathrm{~dB}$ bandwidth-length product, which is determined by the phase shift between the light and the microwave drive signal, was calculated to be 12 GHz for 100 percent intensity modulation.

The DC and high frequency characteristics of the modulator were measured using the experimental arrangement shown in Fig. 3. Electrical bias was supplied through a biasing tee and the drive signal was the amplified output of a HP 8350B Sweep Oscillator. Light from the "pigtailed" single-mode output fiber of an Ortel Model SL-620s diode laser was butt coupled to the modulator input and the output from the modulator was focused into a single-mode fiber. The modulated light was detected by a Newport Model 877 avalanche photodiode or by an Ortel Model RSL-25 photodiode. The input fiber was aligned to the waveguide by imaging the modulator output surface onto an IR-sensitive camera connected to a video monitor. When alignment was correct a single intensity peak, with little scattered light, was observed.

DC measurements yielded a half-wave voltage of approximately $30 \mathrm{~V}$, and the modulation bandwidth is of the order of $500 \mathrm{MHz}$, as shown in Fig. 4. Because both of these results differ significantly from the design values, further study has been undertaken. Since no increase in transmitted intensity is observed for bias voltage greater than $30 \mathrm{~V}$ and no polarization effects are observed at the output, it appears that the dominant effect in the modulation is electro-absorption (Franz-Keldish effect) rather than the electro-optic effect.13 Further evidence of this is that the absorption losses in the modulator, with no applied voltage, are much larger than expected. While this may explain the large extinction voltage, the observed frequency response characteristics remain unexplained at present.

\section{DETECTOR GEOMETRY AND FABRICATION}

An interdigitated electrode geometry with $1 \mu \mathrm{m}$ dimensions (Figure 5 ) was chosen for the detectors to ensure short transit distances for photon-generated carriers while also maximizing the active area. Although this geometry results in a higher intrinsic capacitance than that for a straight-gap device, it is not enough to significantly affect device performance.

The detectors were fabricated on the HEMT-type, MBE-grown material that is shown schematically in Fig. 6. Standard photolithography and wet etching techniques were used for the process, and the evaporated AuGe/Ni/Au ohmic contacts were flash annealed in a $700{ }^{\circ} \mathrm{C}$ furnace. As a final processing step the $400-\AA$ cap layer of $n^{+}$GaAlAs was removed.

\section{DETECTOR PERFORMANCE}

The detectors were mounted on an alumina substrate on which a $50 \Omega$ gold microstrip transmission line had been deposited, as shown schematically in Fig. 7 . Gold wire-bonded connections were made between the detectors and the transmission line, and the output was through a SMA end launcher that was in pressure contact with the line.

DC I-V curves were obtained using a curve tracer while the detectors were illuminated by white light through a microscope. A typical example of an I-V curve at two different illumination levels, shown in Fig. 8, demonstrates that the detectors are symmetric devices and that the contacts are ohmic.

Frequency response measurements were made over the range 0.01 to $10 \mathrm{GHz}$ using the experimental arrangement shown schematically in Fig. 9. The detectors were dc biased through a biasing tee and a $50 \Omega$ load was used to impedance match the detector output to the measurement system. Modulated light was from a directly modulated, fiber-pigtailed ortel Model 
TSL-1000 diode laser, and the rf output signal from the detector was amplified and measured using an HP $8566 \mathrm{~A}$ spectrum analyzer.

A typical example of the measured frequency response of a detector is given in $F i g .10$. As shown in the figure, the $3 \mathrm{~dB}$ cutoff frequency is at approximately $185 \mathrm{MHz}$, with the response falling at about $12 \mathrm{~dB} /$ decade from $185 \mathrm{MHz}$ to near $1 \mathrm{GHz}$. Beyond $1 \mathrm{GHz}$ the response falls at the $20 \mathrm{~dB} /$ decade rate expected of a single-pole device. Preliminary measurements on devices with larger geometry (up to $4 \mu \mathrm{m}$ electrode spacing) that were fabricated on the same material show a similar response, with only the overall amplitude being higher. These results suggest that device speed is limited by minority carrier trapping rather than by geometry, by majority carrier mobility, or by saturation velocity. 14

Measurements have also been made of the responsivity at $500 \mathrm{MHz}$ using an Ortel Model SL-620 diode laser as a source. A typical result of the measurement of detector current as a function of average incident optical power is shown in Fig. 11. The responsivity at $500 \mathrm{MHz}$, measured as the slope of this curve in its most linear region, is $0.21 \mathrm{~A} / \mathrm{W}$. Since responsivity has the same frequency dependence as gain, 15 the low frequency responsivity of this device is $2.5 \mathrm{~A} / \mathrm{W}$, giving an external quantum efficiency of 3.81 , which is comparable to that found in GaAs detectors.

\section{SUMMARY AND CONCLUSIONS}

The performance of a waveguide Mach-Zehnder electro-optic modulator and of a set of interdigitated photoconductive detectors is being studied at frequencies to $10 \mathrm{GHz}$. Although modulation of light has been observed up to about $500 \mathrm{MHz}$, neither the half-wave voltage nor the modulation bandwidth are as predicted by a theoretical model. The device exhibits a higher than predicted absorption at the $820 \mathrm{~nm}$ design wavelength and the observed modulation appears to be from electro-absorption rather than from the expected electro-optic effect. A spectroscopic study is presently underway in an effort to understand the observed behavior. The observed performance of the detectors is comparable to that of GaAs-based photoconductive devices of similar geometry. While they did not exhibit an extremely high bandwidth, they did possess usable gain well into the $\mathrm{GHz}$ range. The bandwidth appears to be limited by minority carrier trapping, and a study is presently underway to evaluate this effect.

\section{ACKNOWLEDGEMENT}

This work was conducted under a cooperative research agreement between NASA Lewis Research Center and Case Western Reserve University (NCC3-99). During part of the period of the research, prof. P.C. Claspy was a participant in the NASA/ASEE Summer Faculty Fellowship Program. Portions of the work on the detectors are from a thesis submitted to Case Western Reserve University by S.M. Hill in partial fulfillment of the requirements for the MS degree in Electrical Engineering.

\section{REFERENCES}

1. K.B. Bhasin, G. Anzic, R.R. Kunath, and D.J. Connolly, "Optical Techniques to Feed and Control GaAs MMIC Modules for Phased Array Antenna Applications," 11th AIAA Communications Satellite Systems Conference, AIAA, New York, 506-514 (1986).

2. K.B. Bhasin and D.J. Connolly, "Advances in Gallium Arsenide Monolithic Microwave Integrated-Circuit Technology for Space Communications Systems," IEEE Trans. Microwave Theory Tech., 34(10), 994-1001 (1986).

3. K.Y. Lau, and A. Yariv, "Ultra-High Speed Semiconductor Lasers," IEEE J. Quantum Electron., 21(2), 121-138 (1985)

4. For example, Ortel Corp., Alhambra, CA.

5. C.M. Gee and G.D. Thurmond, "Wideband Traveling-Wave Electro-optic Modulator," in Optical Technology for Microwave Applications, S.K. Yao, ed., Proc. SPIE Vol. 477, 17-22 $(1984)$

6. P. Buchmann, H. Kaufman, H. Melchior, and G. Guekos, "Broadband Y-Branch Electro-Optic GaAs Waveguide Interferometer for 1.3 $\mu \mathrm{m}, "$ Appl. Phys. Lett., 46(5), 462-464 (1985).

7. S.Y. Wang and D.M. Bloom, "100 GHz Bandwidth Planar GaAs Schottky Photodiode," Electron. Lett., 19(14), 554-555 (1983).

8. C.Y. Chen, A.Y. Cho, C.G. Bethea, P.A. Garbinski, Y.M. Pang, B.F. Levine, and K. Ogawa, "Ultrahigh Speed Modulation-Doped Heterostructure Field-Effect Photodetectors," Appl. Phys. Lett. . 42(12), 1040-1042 (1983).

9. T. Umeda, Y. Cho, and A. Shibatomi, "Picosecond HEMT Photodetector," Japan. J. Appl. Phys. Lett. , 25(10), L801-L803 (1986).

10. D.M. Materna, "Investigation of a GaAlAs Mach-Zehnder Electro-optic Modulator," M.S. Thesis, Case-Western Reserve University, Cleveland, OH, 1986. (NASA CR-179573).

11. D.M. Materna, A.M. Ferendeci, and K.B. Bhasin, "Design of a GaAlAs Traveling Wave Mach-Zehnder Electro-Optic Modulator," in Optoelectronic Materials, Devices, Packaging, and Interconnects, T.E. Batchman, R.F. Carson, R.L. Gallawa, and H.J. Wojtunik, eds., Proc. SPIE VOI. 836, 195-198 (1987). 
12. C.M. Chorey, A. Ferendeci, and K. Bhasin, "A High Frequency GaAlAs Traveling Wave Electro-Optic Modulator at $0.82 \mu \mathrm{m}$," in IEEE MTT-S International Microwave Symposium Digest. Vol. 2, IEEE, Piscataway, NJ, 735-738 (1988).

13. F.K. Reinhart, "Electroabsorption in $\mathrm{Al}_{Y} \mathrm{Ga}_{1}-\mathrm{Y}^{\mathrm{As}-\mathrm{Al}} \mathrm{X}_{\mathrm{X}} \mathrm{Ga}_{1-\mathrm{X}} \mathrm{As}$ Double Heterostructures," Appl. Phys. Lett., 22(8), 372-374 (1973).

14. H. Beneking, "On the Response Behavior of Fast Photoconductive Optical Planar and Coaxial Semiconductor Detectors," IEEE Trans. Electron Devices, 29(9), 1431-1441 (1982).

15. G.J. Papaioannou and J.R. Forrest, "On the Photoresponse of GaAs MESFET's: Backgating and Deep Traps Effect," IEEE Trans. Electron Devices, 33(3), 373-378 (1986).

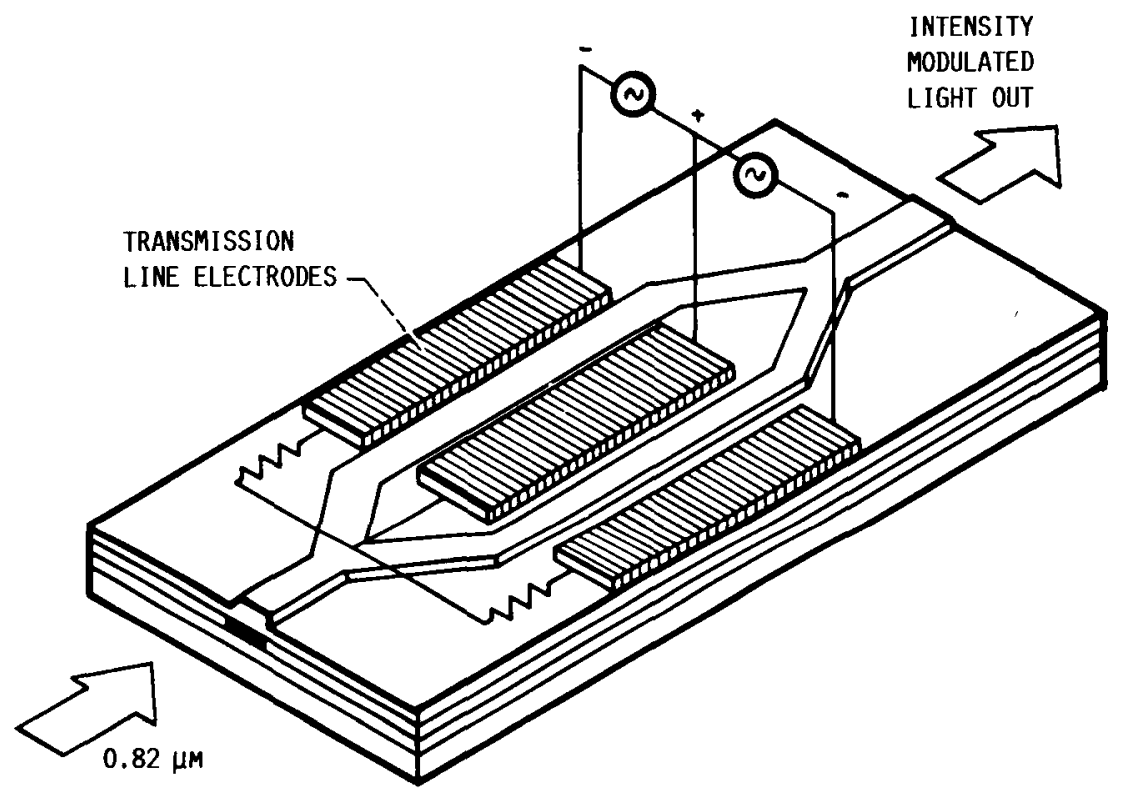

FIG. 1. SCHEMATIC OF MACH-ZEHNDER ELECTROOPTIC MODULATOR.

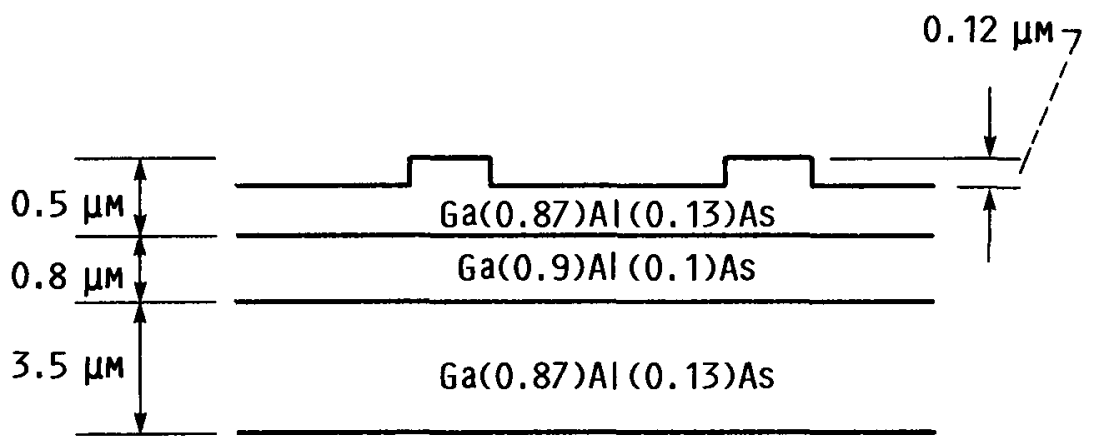

FIG. 2. ELECTROOPTIC MODULATOR MATERIAL SCHEMATIC. 


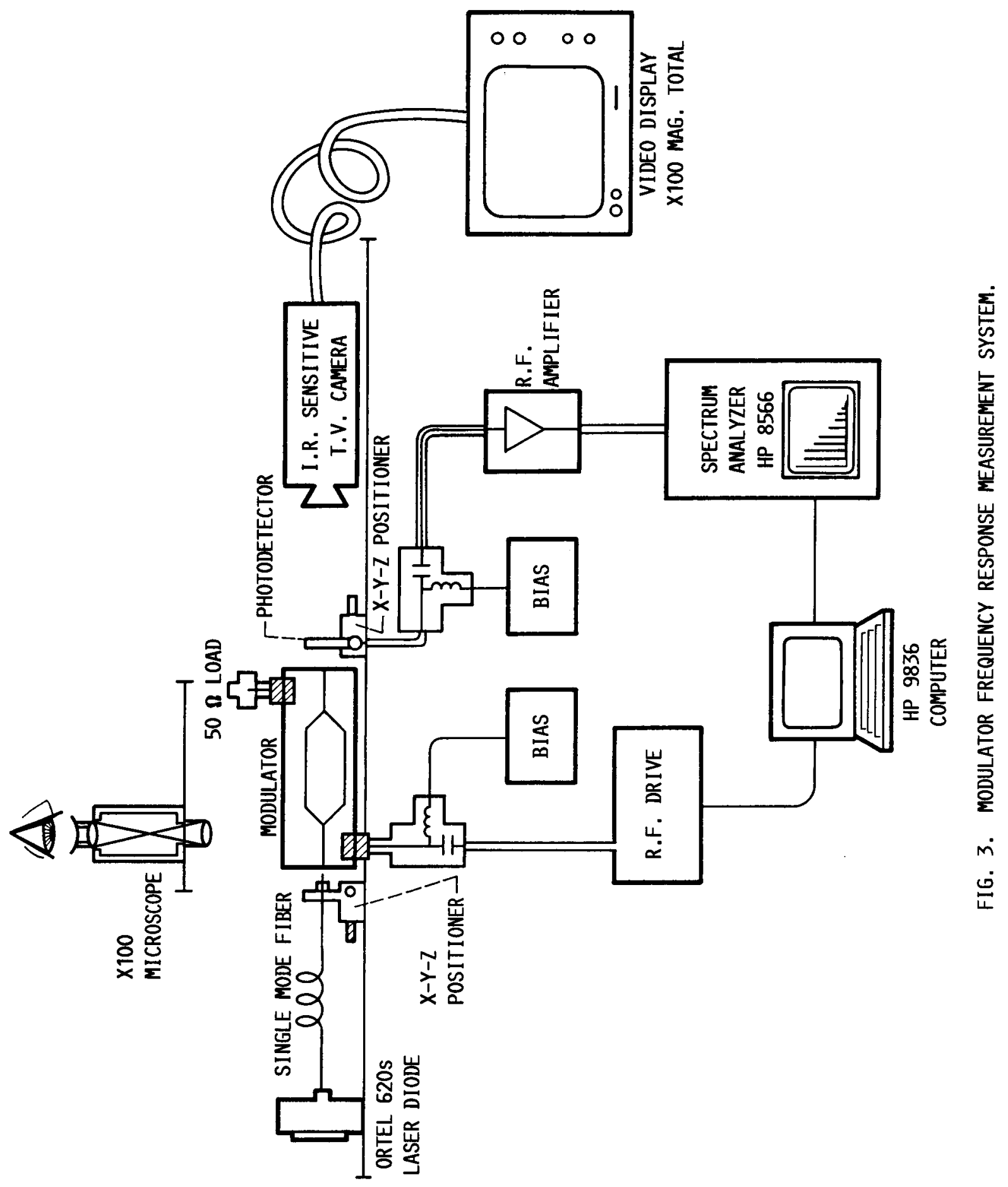




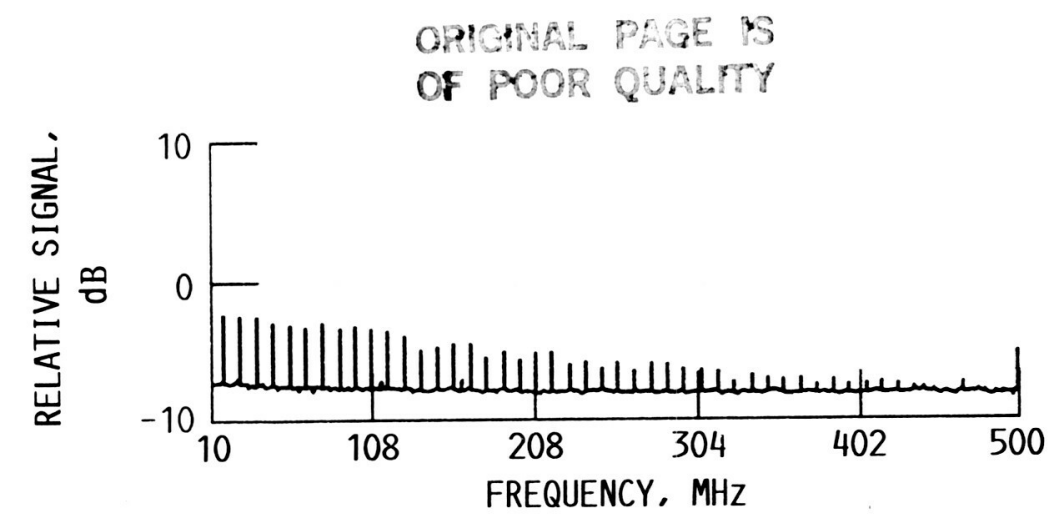

FIG. 4. MODULATOR FREQUENCY RESPONSE BETWEEN 10 AND $500 \mathrm{MHz}$.

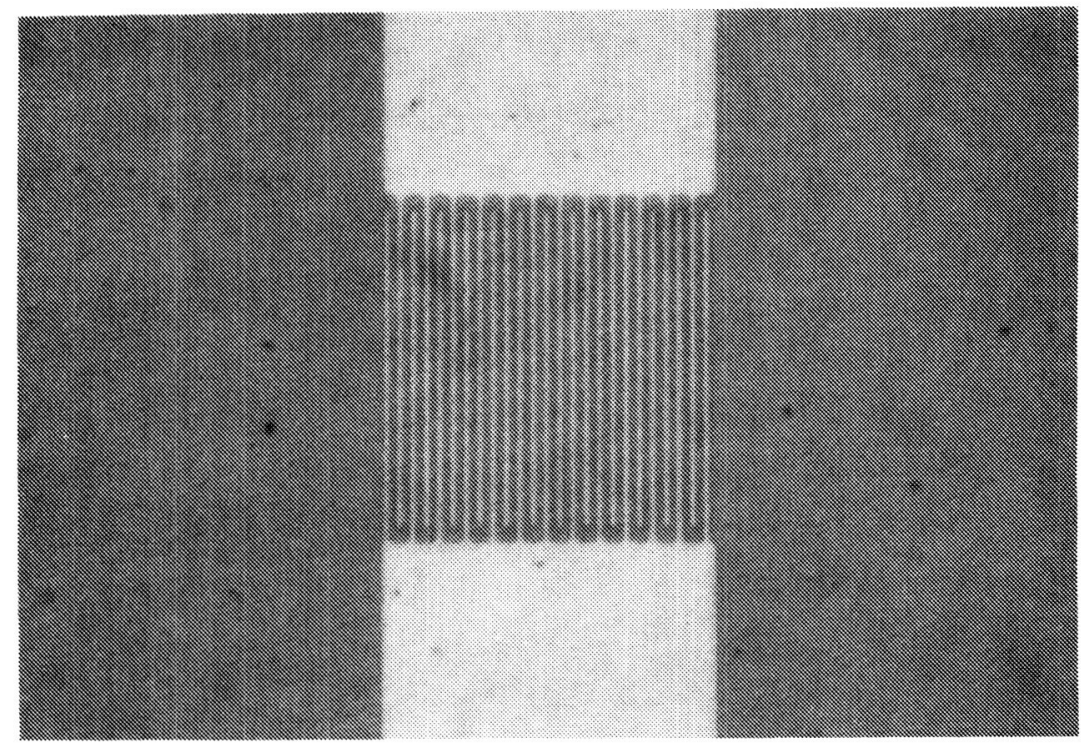

FIG. 5. $1 \mu \mathrm{M} \times 1 \mu \mathrm{M}$ DETECTOR. 


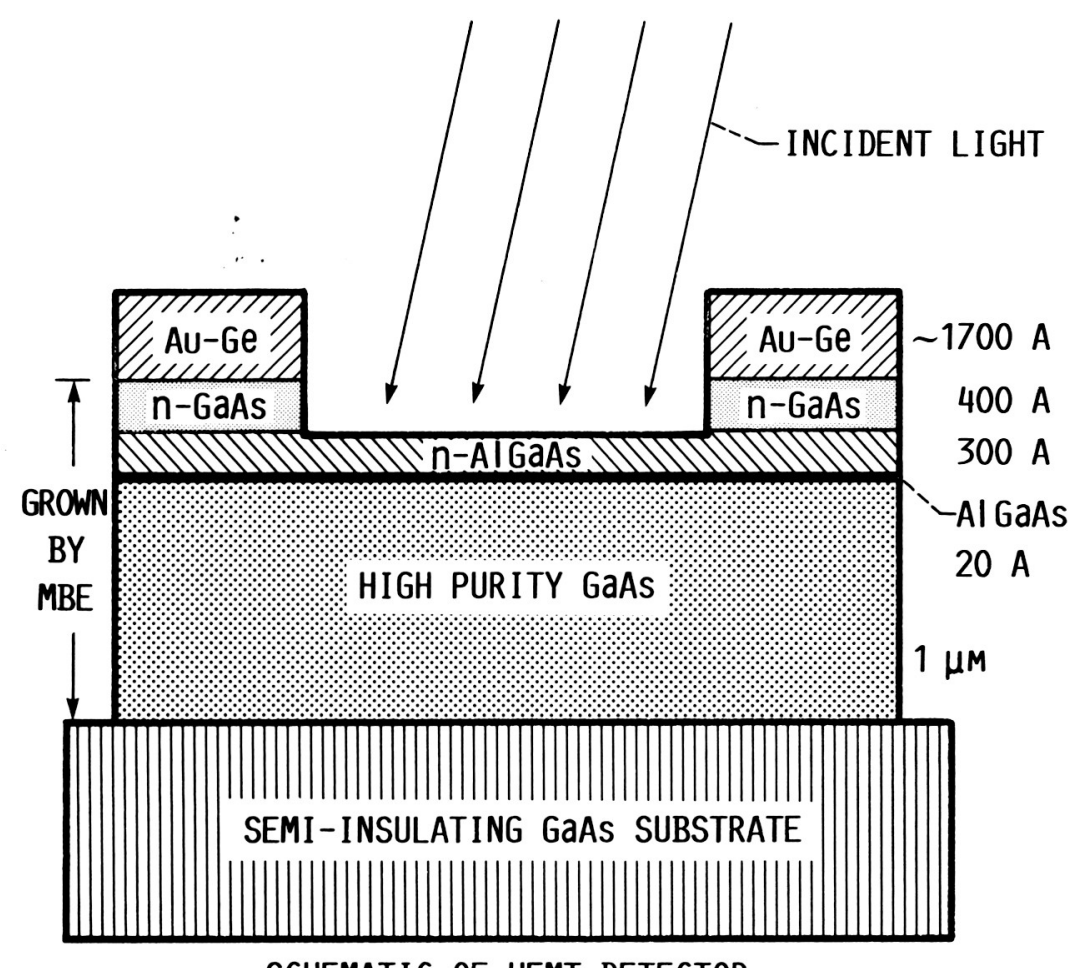

SCHEMATIC OF HEMT DETECTOR

FIG. 6. DETECTOR MATERIAL STRUCTURE.

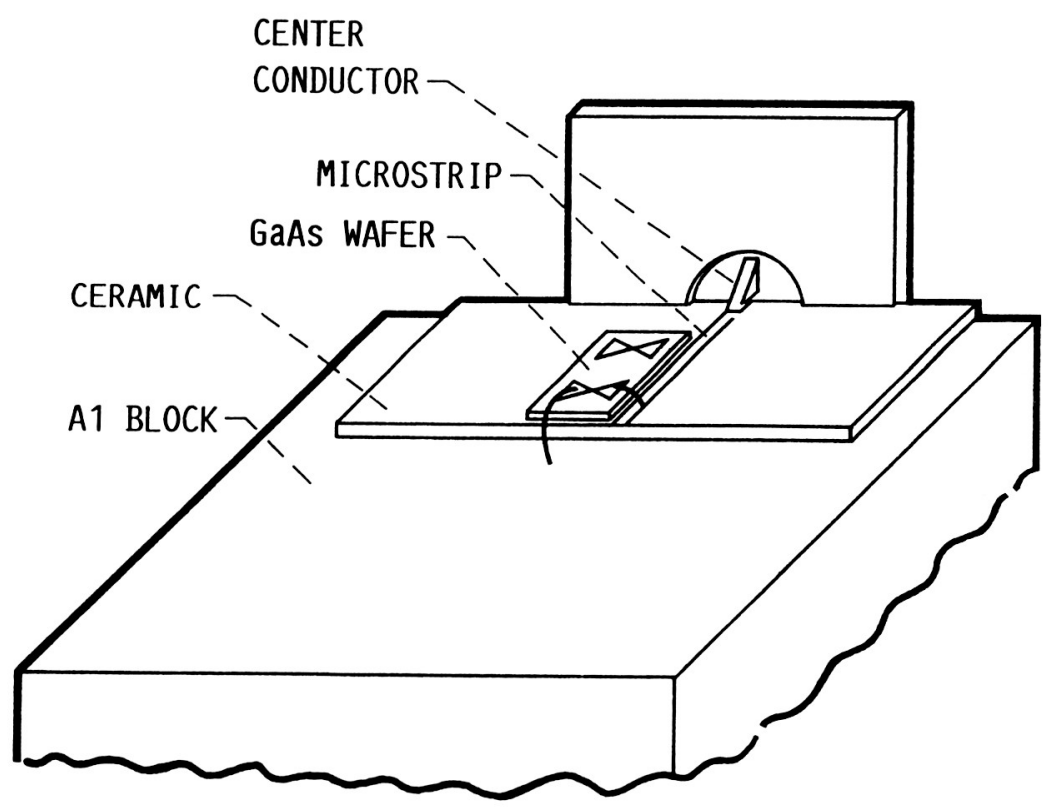

FIG. 7. DETECTOR MOUNT CONFIGURATION. 

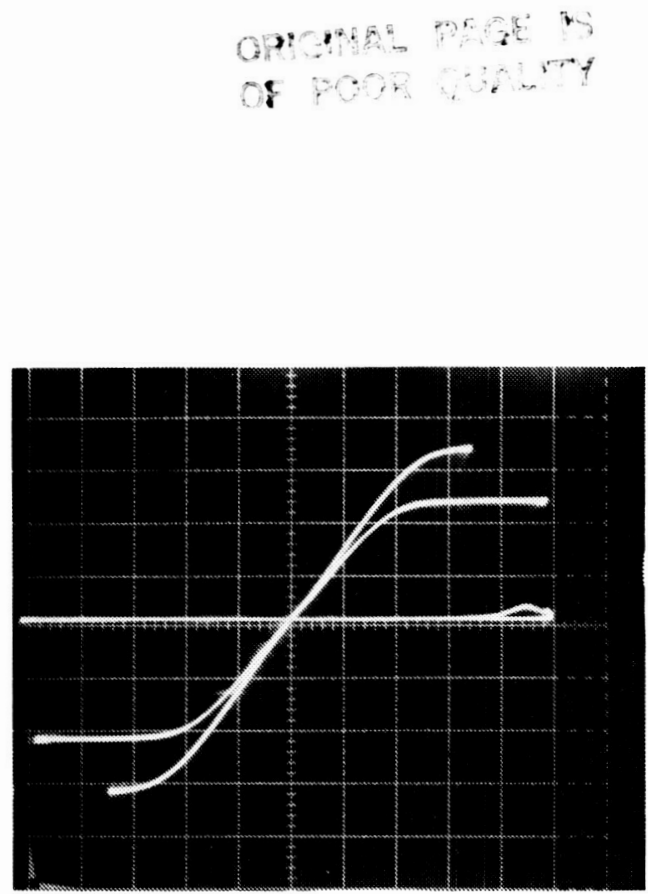

FIG. 8. I-V CHARACTERISTIC OF $1 \mu \mathrm{M} \times 1 \mu \mathrm{M}$ DETECTOR AT THREE DIFFERENT ILLUMINATION LEVELS. 


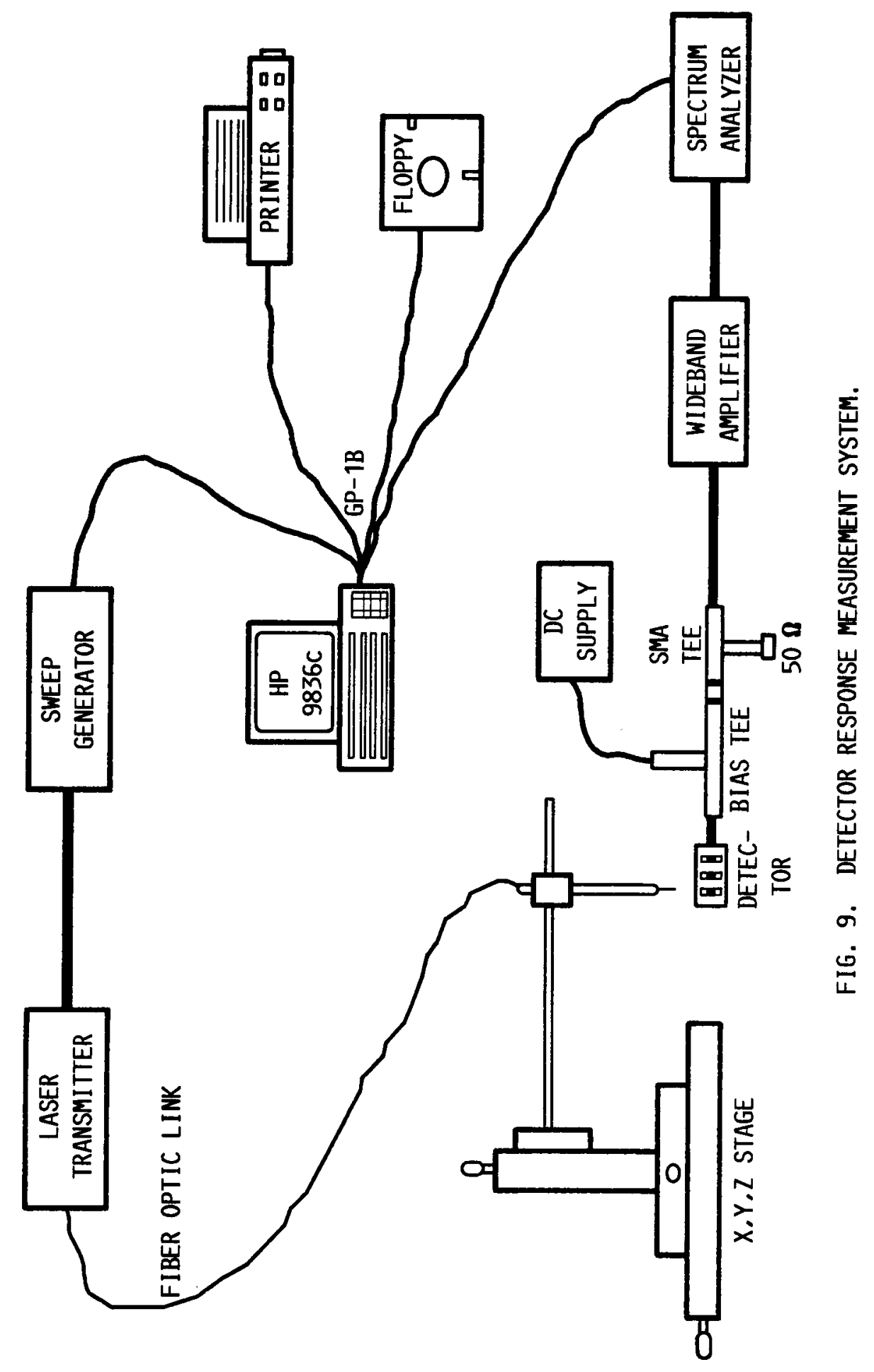




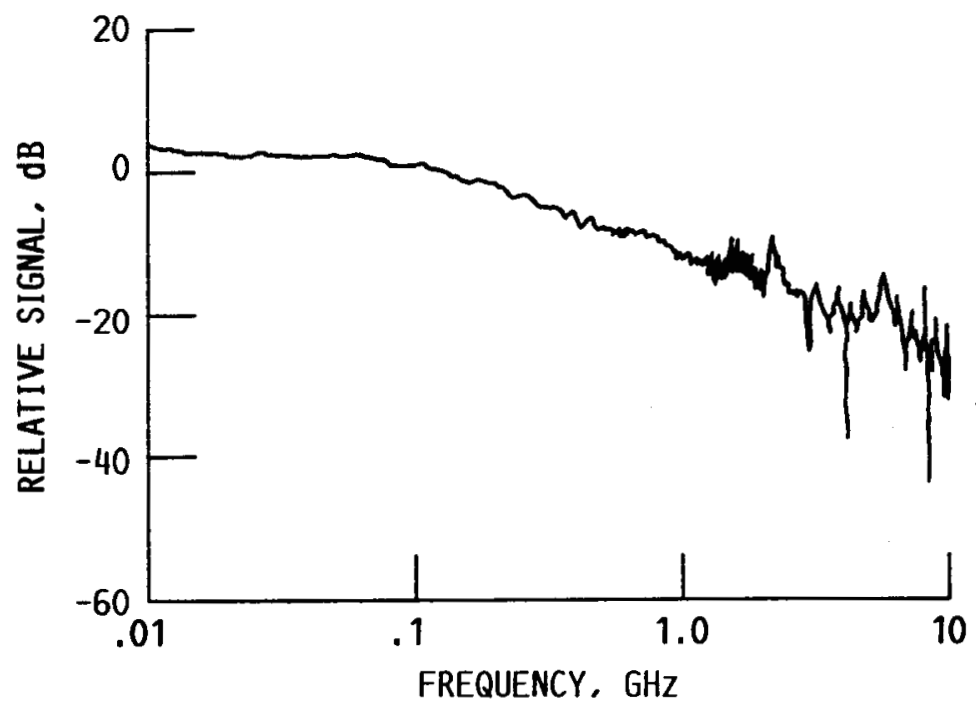

FIG. 10. FREQUENCY RESPONSE OF $1 \mu \mathrm{M} \times 1 \mu \mathrm{M}$ DETECTOR. 


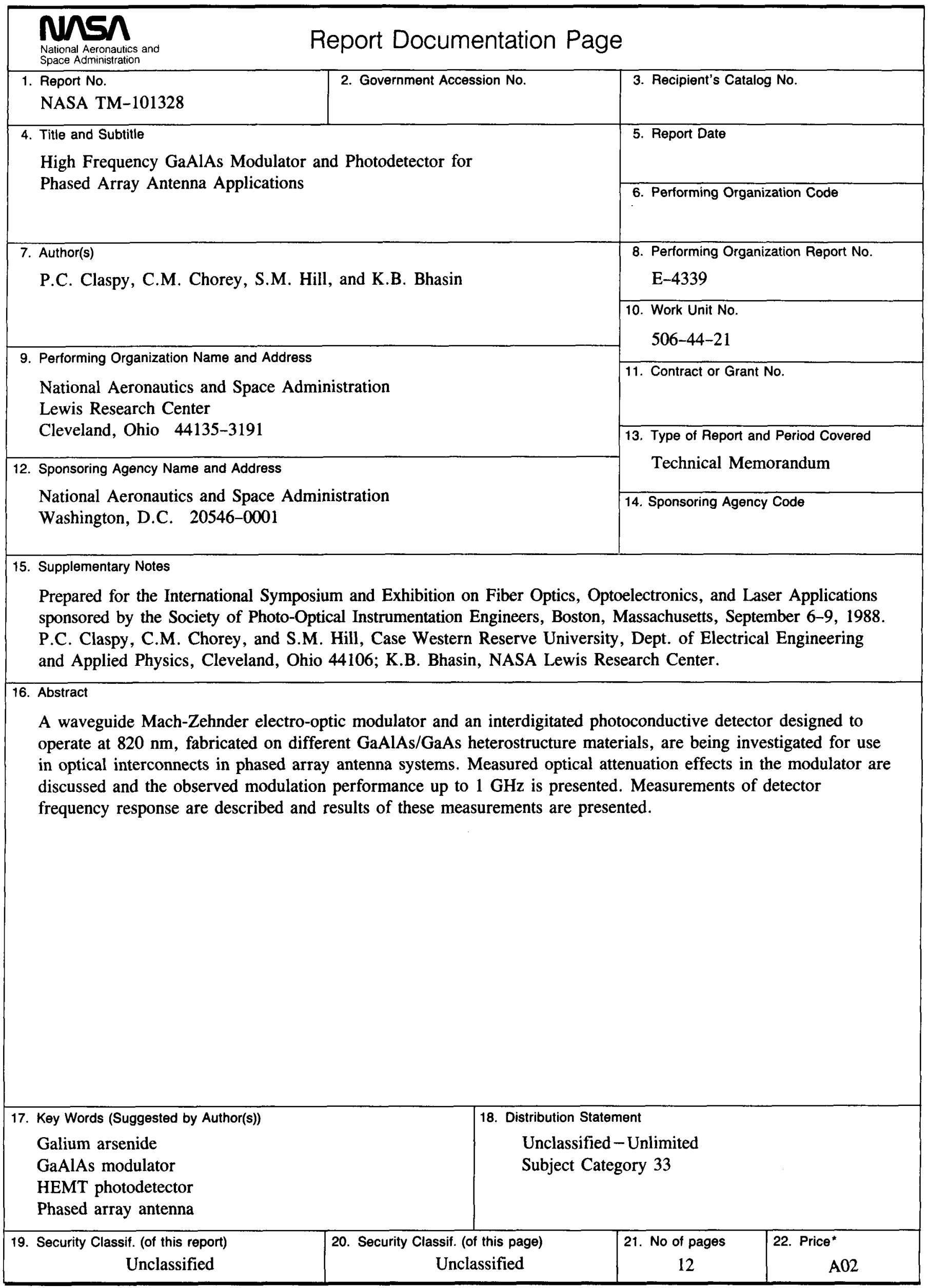

